\title{
Periodic solutions of hybrid jump diffusion processes
}

\author{
Xiao-Xia Guo \\ School of Applied Mathematics \\ Shanxi University of Finance and Economics \\ Taiyuan, 030006, China \\ and \\ School of Mathematics and Information Sciences \\ Guangzhou University \\ Guangzhou, 510006, China \\ E-mail: xxguo91@163.com \\ Wei Sun \\ Department of Mathematics and Statistics \\ Concordia University \\ Montreal, H3G 1M8, Canada \\ E-mail: wei.sun@concordia.ca
}

\begin{abstract}
In this paper, we investigate periodic solutions of regime-switching jump diffusions. We first show the well-posedness of solutions to SDEs corresponding to the hybrid system. Then, we derive the strong Feller property and the irreducibility of the associated timeinhomogeneous semigroups. Finally, we establish the existence and uniqueness of periodic solutions. Concrete examples are presented to illustrate the results.
\end{abstract}

MSC: 60J25; 60H10; 60J75; 34C25

Keywords: Hybrid system; regime-switching jump diffusion; periodic solution; strong Feller property; irreducibility.

\section{Introduction}

A hybrid system is a dynamical system whose evolution depends on both continuous and discrete variables. The study of hybrid systems is becoming more and more important in different research 
areas such as biology, ecosystems, wireless communications, signal processing, engineering and mathematical finance. In the recent years, lots of progress has been made on a class of hybrid systems called regime-switching jump diffusion processes. This model consists of two component processes $(X(t), \Lambda(t))$ with $X(t)$ and $\Lambda(t)$ being of continuous and discrete states, respectively. If $\Lambda(t)$ is a Markov chain that is independent of $X(t)$, then we have the model of Markov-switching jump diffusions; whereas if $\Lambda(t)$ depends on $X(t)$, then we have the model of regime-switching jump diffusions. We refer the reader to the monographs [8, 17] for comprehensive studies of hybrid switching diffusions and their applications.

The model of regime-switching jump diffusions provides more flexibility in applications but also requires careful examination of the dependence between the continuous and discrete components. In the past decade, various properties of these hybrid systems have been thoroughly studied and many remarkable results have been obtained. For example, Chen et al. obtained maximum principles and Harnack inequalities in [2] and discussed the recurrence and ergodicity in [3]. Xi [13], Xi and Yin [14] investigated asymptotic properties of the model. Nguyen and Yin [10, 11], Shao [12], Xi and Zhu [16], Xi, Yin and Zhu [15] considered the model whose switching component has a countably infinite state space. Note that most of the existing literatures have focused on the time-homogeneous case. In this paper, we will study time-inhomogeneous regime-switching jump diffusions and investigate their periodic solutions.

Periodic solutions play an important role in the study of stochastic dynamical systems. Here we just list some previous works that are closely related to this paper. Khasminskii [6] systematically developed the theory of periodic solutions to random systems modelled by stochastic differential equations (SDEs). In [18], Zhang et al. investigated periodic solutions of SDEs driven by Lévy processes. $\mathrm{Hu}$ and $\mathrm{Xu}[5]$ obtained the existence and uniqueness of periodic solutions for stochastic functional differential equations by proving the global attractivity of solutions. In our recent work [4], we established the ergodicity and uniqueness of periodic solutions for jump diffusions by proving the strong Feller property and the irreducibility of the associated time-inhomogeneous semigroups. Note that none of the above mentioned works discusses periodic solutions of hybrid systems. Our present paper seems to be the first one discussing periodic solutions of regimeswitching jump diffusions whose switching components can have countably infinite state spaces.

The rest of this paper is organized as follows. In Section 2, we will show the well-posedness of solutions to SDEs corresponding to the hybrid system (2.1) and (2.2) (see below). The unique strong solution is obtained by representing the switching component as a stochastic integral with respect to a Poisson random measure (see (2.3) below) and by using an interlacing procedure. Different from [14, Proposition 2.1], we only assume that the coefficient functions satisfy the local Lipschitz condition. Also, we remove a key assumption of [12, 15, 16], which requires that the transition matrix of the switching component is Hölder continuous. In Section 3, we will establish the existence and uniqueness of periodic solutions. To this end, we derive the strong Feller property and the irreducibility of the associated time-inhomogeneous semigroups. Our assumption $\mathrm{Q}_{1}$ (see below) is weaker than [12, (A1)], [15, Assumption 5.1] and [16, Assumption 4.3]. Finally, we will give two examples in Section 4 to demonstrate our main results. 


\section{Existence and uniqueness of regime-switching jump dif- fusion processes}

Let $\left(\Omega, \mathcal{F},\left\{\mathcal{F}_{t}\right\}_{t \geq 0}, \mathbb{P}\right)$ be a complete probability space with filtration $\left\{\mathcal{F}_{t}\right\}_{t \geq 0}$ satisfying the usual conditions (i.e., it is increasing, right continuous and $\mathcal{F}_{0}$ contains all $\mathbb{P}$-null sets). Suppose that $k, l, m \in \mathbb{N}$ with $k \geq m$. Denote by $\mathbb{R}_{+}$the set of all non-negative real numbers. Let $\{B(t)\}_{t \geq 0}$ be a $k$-dimensional standard Brownian motion and $N$ be an independent Poisson random measure (corresponding to a stationary point process $p(t))$ on $\mathbb{R}_{+} \times\left(\mathbb{R}^{l}-\{0\}\right)$. The compensator $\widetilde{N}$ of $N$ is given by $\widetilde{N}(\mathrm{~d} t, \mathrm{~d} u)=N(\mathrm{~d} t, \mathrm{~d} u)-\nu(\mathrm{d} u) \mathrm{d} t$, where $\nu(\cdot)$ is a Lévy measure satisfying $\int_{\mathbb{R}^{l}-\{0\}}\left(1 \wedge|u|^{2}\right) \nu(\mathrm{d} u)<\infty$. Let $\mathcal{S}=\{1,2, \ldots\}$.

Throughout this paper, we fix a $\theta>0$. Let $(X(t), \Lambda(t))$ be a pair of right continuous processes with left-hand limits on $\mathbb{R}^{m} \times \mathcal{S}$. The first component $X(t)$ satisfies the following SDE:

$$
\begin{aligned}
d X(t)= & b(t, X(t), \Lambda(t)) \mathrm{d} t+\sigma(t, X(t), \Lambda(t)) \mathrm{d} B(t)+\int_{\{|u|<1\}} H(t, X(t-), \Lambda(t-), u) \widetilde{N}(\mathrm{~d} t, \mathrm{~d} u) \\
& +\int_{\{|u| \geq 1\}} G(t, X(t-), \Lambda(t-), u) N(\mathrm{~d} t, \mathrm{~d} u) .
\end{aligned}
$$

We assume that the coefficient functions $b(t, x, i):[0, \infty) \times \mathbb{R}^{m} \times \mathcal{S} \rightarrow \mathbb{R}^{m}, \sigma(t, x, i):[0, \infty) \times \mathbb{R}^{m} \times$ $\mathcal{S} \rightarrow \mathbb{R}^{m \times k}, H(t, x, i, u):[0, \infty) \times \mathbb{R}^{m} \times \mathcal{S} \times \mathbb{R}^{l} \rightarrow \mathbb{R}^{m}$ and $G(t, x, i, u):[0, \infty) \times \mathbb{R}^{m} \times \mathcal{S} \times \mathbb{R}^{l} \rightarrow \mathbb{R}^{m}$ are all Borel measurable and satisfy

$$
\begin{aligned}
& b(t+\theta, x, i)=b(t, x, i), \quad \sigma(t+\theta, x, i)=\sigma(t, x, i), \\
& H(t+\theta, x, i, u)=H(t, x, i, u), \quad G(t+\theta, x, i, u)=G(t, x, i, u)
\end{aligned}
$$

for any $t \geq 0, x \in \mathbb{R}^{m}, i \in \mathcal{S}$ and $u \in \mathbb{R}^{l}-\{0\}$. The second component $\Lambda(t)$ has the state space $\mathcal{S}$ such that

$$
\mathbb{P}\{\Lambda(t+\Delta)=j \mid \Lambda(t)=i, X(t)=x\}= \begin{cases}q_{i j}(x) \Delta+o(\Delta), & i \neq j, \\ 1+q_{i j}(x) \Delta+o(\Delta), & i=j,\end{cases}
$$

as $\Delta \downarrow 0$. Hereafter $q_{i j}(x)$ is a Borel measurable function on $\mathbb{R}^{m}$ for $i, j \in \mathcal{S}$ such that $q_{i j}(x) \geq 0$ for all $i, j \in \mathcal{S}$ with $i \neq j$ and $\sum_{j \in \mathcal{S}} q_{i j}(x)=0$ for all $i \in \mathcal{S}$ and $x \in \mathbb{R}^{m}$. We make the following assumption on the matrix $Q=\left(q_{i j}(x)\right)$.

$\left.\mathrm{Q}_{1}\right)$

$$
\sum_{j=1}^{\infty} \sup _{i \in \mathcal{S} \backslash\{j\}, x \in \mathbb{R}^{m}} q_{i j}(x)<\infty .
$$

We point out that $\Lambda(t)$ can be represented as a stochastic integral with respect to a Poisson random measure. To this end, we construct a family of intervals $\left\{\Delta_{i j}(x): i, j \in \mathcal{S}, i \neq j\right\}$ on the 
positive real line as follows: for $x \in \mathbb{R}^{m}$ and $i, j \in \mathcal{S}$ with $j \neq i$, set

$$
\begin{aligned}
\Delta_{i 1}(x) & =\left[0, q_{i 1}(x)\right), \\
\Delta_{i 2}(x) & =\left[q_{i 1}(x), q_{i 1}(x)+q_{i 2}(x)\right), \\
\vdots & \\
\Delta_{i j}(x) & =\left[\sum_{s=1}^{j-1} q_{i s}(x), \sum_{s=1}^{j} q_{i s}(x)\right), \\
\vdots &
\end{aligned}
$$

If $q_{i j}(x)=0$ for $j \neq i$, we set $\Delta_{i j}(x)=\emptyset$. Define

$$
a(j):=\sup _{i \in \mathcal{S} \backslash\{j\}, x \in \mathbb{R}^{m}} q_{i j}(x), \quad L=\sum_{j=1}^{\infty} a(j) .
$$

By the assumption $\mathbf{Q}_{1}$, we get $L<\infty$. Then, each value of the interval $\Delta_{i j}(x)$ is bounded by $L$.

We define a function $h: \mathbb{R}^{m} \times \mathcal{S} \times[0, L] \rightarrow \mathbb{R}$ by $h(x, i, r)=\sum_{j \in \mathcal{S}}(j-i) 1_{\Delta_{i j}(x)}(r)$, that is,

$$
h(x, i, r)= \begin{cases}j-i, & \text { if } r \in \Delta_{i j}(x) \\ 0, & \text { otherwise }\end{cases}
$$

Then, $\Lambda(t)$ can be modeled by

$$
d \Lambda(t)=\int_{[0, L]} h(X(t-), \Lambda(t-), r) N_{1}(d t, d r),
$$

where $N_{1}(d t, d r)$ is a Poisson random measure (corresponding to a stationary point process $p_{1}(t)$ which is adapted to $\left.\mathcal{F}_{t}\right)$ with characteristic measure $\mathcal{L}(d r)$, the Lebesgue measure on $[0, L]$. The Poisson random measure $N_{1}(\cdot, \cdot)$ is assumed to be independent of the Brownian motion $B(\cdot)$ and the Poisson random measure $N(\cdot, \cdot)$. Therefore, the processes $(X(t), \Lambda(t))$ modeled by (2.1) and (2.2) can be thought of as a solution to the hybrid system (2.1) and (2.3).

We use $|x|$ to denote the Euclidean norm of a vector $x$, use $A^{T}$ to denote the transpose of a $\operatorname{matrix} A$, and use $|A|:=\sqrt{\operatorname{trace}\left(A^{T} A\right)}$ to denote the trace norm of $A$. Let $C^{1,2}\left(\mathbb{R}_{+} \times \mathbb{R}^{m} \times \mathcal{S} ; \mathbb{R}\right)$ be the space of all real-valued functions $f(t, x, i)$ on $\mathbb{R}_{+} \times \mathbb{R}^{m} \times \mathcal{S}$ which are continuously differentiable with respect to $t$ and twice continuously differentiable with respect to $x$. For $f \in C^{1,2}\left(\mathbb{R}_{+} \times \mathbb{R}^{m} \times\right.$ $\mathcal{S} ; \mathbb{R})$, define

$$
\mathcal{A} f(t, x, i):=\mathcal{L}_{i} f(t, x, i)+Q(x) f(t, x, \cdot)(i),
$$

with

$$
\begin{aligned}
& \mathcal{L}_{i} f(\cdot, \cdot, i)(t, x) \\
:= & f_{t}(t, x, i)+\left\langle f_{x}(t, x, i), b(t, x, i)\right\rangle+\frac{1}{2} \operatorname{trace}\left(\sigma^{T}(t, x, i) f_{x x}(t, x, i) \sigma(t, x, i)\right) \\
& +\int_{\{|u|<1\}}\left[f(t, x+H(t, x, i, u), i)-f(t, x, i)-\left\langle f_{x}(t, x, i), H(t, x, i, u)\right\rangle\right] \nu(\mathrm{d} u) \\
& +\int_{\{|u| \geq 1\}}[f(t, x+G(t, x, u, i), i)-f(t, x, i)] \nu(\mathrm{d} u),
\end{aligned}
$$


and

$$
Q(x) f(t, x, \cdot)(i):=\sum_{j \in \mathcal{S}} q_{i j}(x)[f(t, x, j)-f(t, x, i)]
$$

where $f_{t}=\frac{\partial f}{\partial t}, f_{x}=\nabla_{x} f=\left(\frac{\partial f}{\partial x_{1}}, \ldots, \frac{\partial f}{\partial x_{m}}\right)$ and $f_{x x}=\left(\frac{\partial^{2} f}{\partial x_{i} \partial x_{j}}\right)_{m \times m}$. Define a metric $d(\cdot, \cdot)$ on $\mathbb{R}^{m} \times \mathcal{S}$ by $d((x, i),(y, j))=|x-y|+|i-j|$. A Borel measurable function $g$ on $[0, \infty)$ is said to be locally integrable, denoted by $g \in L_{l o c}^{1}([0, \infty) ; \mathbb{R})$, if

$$
\int_{0}^{\tau}|g(x)| d x<\infty, \quad \forall \tau>0
$$

We make the following assumptions.

A $)$ For each $i \in \mathcal{S}$,

$$
b(\cdot, 0, i), \sigma(\cdot, 0, i) \in L^{2}\left([0, \theta) ; \mathbb{R}^{m}\right), \quad \int_{\{|u|<1\}}|H(\cdot, 0, i, u)|^{2} \nu(\mathrm{d} u) \in L^{1}\left([0, \theta) ; \mathbb{R}^{m}\right) .
$$

For each $n \in \mathbb{N}$, there exists $L_{n}(t) \in L^{1}\left([0, \theta) ; \mathbb{R}_{+}\right)$such that for any $t \in[0, \theta), i \in \mathcal{S}$ and $x, y \in \mathbb{R}^{m}$ with $|x| \vee|y| \leq n$,

$$
\begin{aligned}
& |b(t, x, i)-b(t, y, i)|^{2} \leq L_{n}(t)|x-y|^{2}, \quad|\sigma(t, x, i)-\sigma(t, y, i)|^{2} \leq L_{n}(t)|x-y|^{2}, \\
& \int_{\{|u|<1\}}|H(t, x, i, u)-H(t, y, i, u)|^{2} \nu(\mathrm{d} u) \leq L_{n}(t)|x-y|^{2} .
\end{aligned}
$$

$\left.\mathbf{B}_{1}\right)$ There exist $V_{1} \in C^{1,2}\left(\mathbb{R}_{+} \times \mathbb{R}^{m} ; \mathbb{R}_{+}\right)$and $g_{1} \in L_{\text {loc }}^{1}([0, \infty) ; \mathbb{R})$ such that

$$
\lim _{|x| \rightarrow \infty}\left[\inf _{t \in[0, \infty)} V_{1}(t, x)\right]=\infty,
$$

and for any $t \geq 0, i \in \mathcal{S}$ and $x \in \mathbb{R}^{m}$

$$
\mathcal{L}_{i} V_{1}(t, x) \leq g_{1}(t)
$$

Theorem 2.1 Suppose that assumptions $\mathbf{A}_{\mathbf{1}}, \mathbf{B}_{\mathbf{1}}$ and $\mathbf{Q}_{\mathbf{1}}$ hold. Then, the hybrid system given by (2.1) and (2.3) has a unique strong solution $(X(t), \Lambda(t))$ with initial value $(X(0), \Lambda(0))=(x, i)$. Moreover, $\mathbb{P}\left\{T_{\infty}=\infty\right\}=1$, where $T_{\infty}=\lim _{n \rightarrow \infty} T_{n}$ and $T_{n}=\inf \{t \geq 0:|X(t)| \vee \Lambda(t) \geq n\}$.

Proof. Let $(x, i) \in \mathbb{R}^{m} \times \mathcal{S}$. By [4, Theorem 2.2], under assumptions $\mathbf{A}_{\mathbf{1}}$ and $\mathbf{B}_{\mathbf{1}}$, there exists a unique non-explosive strong solution $X^{(i)}(t)$ to the following SDE:

$$
\begin{aligned}
d X^{(i)}(t)= & b\left(t, X^{(i)}(t), i\right) \mathrm{d} t+\sigma\left(t, X^{(i)}(t), i\right) \mathrm{d} B(t)+\int_{\{|u|<1\}} H\left(t, X^{(i)}(t-), i, u\right) \tilde{N}(\mathrm{~d} t, \mathrm{~d} u) \\
& +\int_{\{|u| \geq 1\}} G\left(t, X^{(i)}(t-), i, u\right) N(\mathrm{~d} t, \mathrm{~d} u)
\end{aligned}
$$


with initial value $X^{(i)}(0)=x$. Let $\sigma_{1}<\sigma_{2}<\cdots<\sigma_{n}<\cdots$ be the set of all jump points of the stationary point process $p_{1}(t)$ corresponding to the Poisson random measure $N_{1}(d t, d r)$. Since $\mathcal{L}(\cdot)$ is a finite measure on $[0, L], \lim _{n \rightarrow \infty} \sigma_{n}=+\infty$ almost surely.

We now construct the processes $(X(t), \Lambda(t))$. Define

$$
(X(t), \Lambda(t))=\left(X^{(i)}(t), i\right), \quad t \in\left[0, \sigma_{1}\right) .
$$

Let

$$
\Lambda\left(\sigma_{1}\right)=i+\sum_{j \in \mathcal{S}}(j-i) 1_{\Delta_{i j}\left(X^{(i)}\left(\sigma_{1}-\right)\right)}\left(p_{1}\left(\sigma_{1}\right)\right)
$$

Define

$$
(X(t), \Lambda(t))=\left(X^{(i)}\left(\sigma_{1}\right), \Lambda\left(\sigma_{1}\right)\right), \quad t=\sigma_{1} .
$$

Note that (2.1) is equivalent to (2.6) on the time interval $\left[0, \sigma_{1}\right)$. Hence the above defined processes $(X(t), \Lambda(t))$ provide the unique strong solution to the hybrid system (2.1) and (2.3) on $\left[0, \sigma_{1}\right)$.

Let

$$
\widetilde{B}(t)=B\left(t+\sigma_{1}\right)-B(t), \quad \widetilde{p}(t)=p\left(t+\sigma_{1}\right), \quad \widetilde{p_{1}}(t)=p_{1}\left(t+\sigma_{1}\right)
$$

Set

$$
\begin{aligned}
& (\widetilde{X}(t), \widetilde{\Lambda}(t))=\left(X^{\left(\Lambda\left(\sigma_{1}\right)\right)}(t), \Lambda\left(\sigma_{1}\right)\right), \quad t \in\left[0, \sigma_{2}-\sigma_{1}\right) \\
& \widetilde{X}\left(\sigma_{2}-\sigma_{1}\right)=X^{\left(\Lambda\left(\sigma_{1}\right)\right)}\left(\sigma_{2}-\sigma_{1}\right), \\
& \widetilde{\Lambda}\left(\sigma_{2}-\sigma_{1}\right)=\Lambda\left(\sigma_{1}\right)+\sum_{j \in \mathcal{S}}\left(j-\Lambda\left(\sigma_{1}\right)\right) 1_{\widetilde{A}(j)}\left(\widetilde{p}_{1}\left(\sigma_{2}-\sigma_{1}\right)\right)
\end{aligned}
$$

where

$$
\widetilde{A}(j)=\Delta_{\Lambda\left(\sigma_{1}\right), j}\left(X^{\left(\Lambda\left(\sigma_{1}\right)\right)}\left(\left(\sigma_{2}-\sigma_{1}\right)-\right)\right)
$$

Then, we define

$$
(X(t), \Lambda(t))=\left(\widetilde{X}\left(t-\sigma_{1}\right), \widetilde{\Lambda}\left(t-\sigma_{1}\right)\right), \quad t \in\left[\sigma_{1}, \sigma_{2}\right]
$$

which together with (2.7) and (2.8) gives the unique strong solution on the time interval $\left[0, \sigma_{2}\right]$. Continuing this procedure inductively, we define $(X(t), \Lambda(t))$ on the time interval $\left[0, \sigma_{n}\right]$ for each $n$. Therefore, $(X(t), \Lambda(t))$ is the unique strong solution to the hybrid system (2.1) and (2.3).

Next, we show that $(X(t), \Lambda(t))$ is non-explosive. Define

$$
\tau_{0}=0, \quad \tau_{p}=\inf \left\{t>\tau_{p-1}: \Lambda(t) \neq \Lambda\left(\tau_{p-1}\right)\right\}, \quad \tau=\lim _{p \rightarrow \infty} \tau_{p}
$$

Then, $\tau$ is the first instant prior to which $\Lambda(t)$ has infinitely many switches. Note that $\left\{\tau_{p}\right\}_{p \geq 1}$ is a subsequence of $\left\{\sigma_{n}\right\}_{n \geq 1}$ since $\Lambda(t)$ can have jumps only at the time sequence $\left\{\sigma_{n}\right\}_{n \geq 1}$. Define $q_{i}(x):=-q_{i i}(x)$ for $x \in \mathbb{R}^{m}$ and $i \in \mathcal{S}$. Based on the interlacing construction of $(X(t), \Lambda(t))$, we get

$$
\mathbb{P}\left\{\tau_{p+1}-\tau_{p}>t\right\}=\mathbb{E}\left[\exp \left\{-\int_{0}^{t} q_{\Lambda\left(\tau_{p}\right)}\left(X^{\Lambda\left(\tau_{p}\right)}(s)\right) d s\right\}\right]
$$


and

$$
\begin{aligned}
\mathbb{P}\left\{\Lambda\left(\tau_{p+1}\right)=j \mid \mathcal{F}_{\tau_{p+1}-}\right\}= & \frac{q_{\Lambda\left(\tau_{p}\right), j}\left(X\left(\tau_{p+1}-\right)\right)}{q_{\Lambda\left(\tau_{p}\right)}\left(X\left(\tau_{p+1}-\right)\right)}\left(1-\delta_{\Lambda\left(\tau_{p}\right), j}\right) 1_{\left\{q_{\Lambda\left(\tau_{p}\right)}\left(X\left(\tau_{p+1}-\right)\right)>0\right\}} \\
& +\delta_{\Lambda\left(\tau_{p}\right), j} 1_{\left\{q_{\Lambda\left(\tau_{p}\right)}\left(X\left(\tau_{p+1}-\right)\right)=0\right\}} .
\end{aligned}
$$

By the assumption $\mathbf{Q}_{1}$, for any $i \in \mathcal{S}$, we have

$$
q_{i}(x)=-q_{i i}(x)=\sum_{j \in \mathcal{S} \backslash\{i\}} q_{i j}(x) \leq \sum_{j=1}^{\infty} a(j)=L .
$$

Then, (2.9) implies that $\mathbb{P}\left\{\tau_{p+1}-\tau_{p}>t\right\} \geq e^{-L t}$ for all $p \in \mathbb{N}$ and $t>0$. Hence

$$
\begin{aligned}
\mathbb{P}\left\{\tau_{\infty}=\infty\right\} & \geq \mathbb{P}\left\{\left\{\tau_{p+1}-\tau_{p}>t\right\} \text { i.o. }\right\} \\
& =\mathbb{P}\left\{\bigcap_{r=1}^{\infty} \bigcup_{s=r}^{\infty}\left\{\tau_{s+1}-\tau_{s}>t\right\}\right\} \\
& =\lim _{r \rightarrow \infty} \mathbb{P}\left\{\bigcup_{s=r}^{\infty}\left\{\tau_{s+1}-\tau_{s}>t\right\}\right\} \\
& \geq \limsup _{r \rightarrow \infty} \mathbb{P}\left\{\tau_{r+1}-\tau_{r}>t\right\} \\
& \geq e^{-L t} .
\end{aligned}
$$

Letting $t \downarrow 0$, we get $\mathbb{P}\left\{\tau_{\infty}=\infty\right\}=1$. Therefore, $(X(t), \Lambda(t))$ is the unique strong solution to the hybrid system (2.1) and (2.3) with initial value $(x, i)$ for all $t \in[0, \infty)$.

Finally, we show that $\mathbb{P}\left\{T_{\infty}=\infty\right\}=1$. If this is not true, then there exist $\varepsilon>0$ and $T^{\prime} \in(0, \infty)$ such that

$$
\mathbb{P}\left\{T_{\infty} \leq T^{\prime}\right\}>2 \varepsilon .
$$

Hence we can find a sufficiently large integer $n_{0}$ such that

$$
\mathbb{P}\left\{T_{n} \leq T^{\prime}\right\}>\varepsilon, \quad \forall n \geq n_{0} .
$$

Define an increasing sequence by

$$
\rho_{1}=1, \quad \rho_{n}=\min \left\{r \in \mathbb{N}: r \geq \rho_{n-1}+2, \quad \sum_{j=r}^{\infty} a(j) \leq \frac{1}{2^{n}}\right\} .
$$

Set $f(j)=n$ for $j \in\left[\rho_{n}, \rho_{n+1}\right)$ and $n \in \mathbb{N}$. Then, $f$ is a non-decreasing function on $\mathcal{S}$ satisfying $f(j) \rightarrow \infty$ as $j \rightarrow \infty$ and

$$
\sum_{j=1}^{\infty} a(j) f(j)=\sum_{n=1}^{\infty} \sum_{j \in\left[\rho_{n}, \rho_{n+1}\right)} a(j) f(j) \leq \sum_{n=1}^{\infty} \frac{n}{2^{n}}<\infty .
$$


Define $V(t, y, k):=V_{1}(t, y)+f(k)$, where $V_{1}$ is given in the assumption $\mathbf{B}_{1}$. By Itô's formula, (2.5) and the assumption $\mathbf{Q}_{1}$, we get

$$
\begin{aligned}
& \mathbb{E}_{0, x, i}\left[V\left(t \wedge T_{n}, X\left(t \wedge T_{n}\right), \Lambda\left(t \wedge T_{n}\right)\right)\right] \\
= & V(0, X(0), \Lambda(0))+\mathbb{E}_{0, x, i}\left[\int_{0}^{t \wedge T_{n}} \mathcal{A} V(s, X(s), \Lambda(s)) d s\right] \\
= & V(0, x, i)+\mathbb{E}_{0, x, i}\left[\int_{0}^{t \wedge T_{n}} \mathcal{L}_{\Lambda(s)} V_{1}(s, X(s)) d s\right] \\
& +\mathbb{E}_{0, x, i}\left[\int_{0}^{t \wedge T_{n}} \sum_{j \in \mathcal{S}} q_{\Lambda(s), j}(X(s))[f(j)-f(\Lambda(s))] d s\right] \\
\leq & V(0, x, i)+\mathbb{E}_{0, x, i}\left[\int_{0}^{t \wedge T_{n}} g_{1}(s) d s\right]+\mathbb{E}_{0, x, i}\left[\int_{0}^{t \wedge T_{n}} \sum_{j=1}^{\infty} a(j) f(j) d s\right] .
\end{aligned}
$$

Then, we have

$$
\mathbb{E}_{0, x, i}\left[V\left(T^{\prime} \wedge T_{n}, X\left(T^{\prime} \wedge T_{n}\right), \Lambda\left(T^{\prime} \wedge T_{n}\right)\right)\right] \leq V(0, x, i)+\int_{0}^{T^{\prime}} g_{1}(s) d s+T^{\prime} \sum_{j=1}^{\infty} a(j) f(j) .
$$

Set

$$
M:=V(0, x, i)+\int_{0}^{T^{\prime}} g_{1}(s) d s+T^{\prime} \sum_{j=1}^{\infty} a(j) f(j)<\infty .
$$

Then,

$$
\mathbb{E}_{0, x, i}\left[1_{\left\{T_{n} \leq T^{\prime}\right\}} V\left(T_{n}, X\left(T_{n}\right), \Lambda\left(T_{n}\right)\right)\right] \leq M
$$

Define

$$
\mu(n)=\inf \left\{V(t, y, j):(t, y, j) \in \mathbb{R}_{+} \times \mathbb{R}^{m} \times \mathcal{S},|y| \vee j \geq n\right\} .
$$

Then, $\lim _{n \rightarrow \infty} \mu(n)=\infty$ by (2.4) and our choice of $f$. However, by (2.10) and (2.11), it follows that

$$
\varepsilon \mu(n)<\mu(n) \mathbb{P}\left\{T_{n} \leq T^{\prime}\right\} \leq M .
$$

We have arrived at a contradiction. Therefore,

$$
T_{\infty}=\infty \quad \text { a.s. }
$$

The proof is complete.

\section{Periodic solutions of regime-switching jump diffusion processes}

In this section, we will study the existence and uniqueness of periodic solutions of the hybrid system given by (2.1) and (2.3). Denote by $\mathcal{B}\left(\mathbb{R}^{m} \times \mathcal{S}\right)$ the Borel $\sigma$-algebra of $\mathbb{R}^{m} \times \mathcal{S}$ and denote 
by $B_{b}\left(\mathbb{R}^{m} \times \mathcal{S}\right)$ (respectively $\left.C_{b}\left(\mathbb{R}^{m} \times \mathcal{S}\right)\right)$ the space of all real-valued bounded Borel functions (respectively continuous and bounded functions) on $\mathbb{R}^{m} \times \mathcal{S}$. Suppose that $\{Y(t), t \geq 0\}$ is a Markov process on $\mathbb{R}^{m} \times \mathcal{S}$. We define its transition probability function by

$$
P(s, y, t, A)=\mathbb{P}\{Y(t) \in A \mid Y(s)=y\}, \quad y \in \mathbb{R}^{m} \times \mathcal{S}, 0 \leq s<t,
$$

and define the corresponding Markovian semigroup of linear operators $\left\{P_{s, t}\right\}$ on $B_{b}\left(\mathbb{R}^{m} \times \mathcal{S}\right)$ by

$$
\left(P_{s, t} f\right)(y):=\mathbb{E}_{s, y}[f(Y(t))]:=\int_{\mathbb{R}^{m} \times \mathcal{S}} f(z) P(s, y, t, d z), \quad y \in \mathbb{R}^{m} \times \mathcal{S}, 0 \leq s<t .
$$

Definition 3.1 (i) A Markov process $\{Y(t), t \geq 0\}$ on $\mathbb{R}^{m} \times \mathcal{S}$ is said to be $\theta$-periodic if for any $n \in \mathbb{N}$ and any $0 \leq t_{1}<t_{2}<\cdots<t_{n}$, the joint distribution of the random variables $Y\left(t_{1}+k \theta\right), Y\left(t_{2}+k \theta\right), \ldots, Y\left(t_{n}+k \theta\right)$ is independent of $k$ for $k \in \mathbb{N} \cup\{0\}$. A Markovian transition semigroup $\left\{P_{s, t}\right\}$ is said to be $\theta$-periodic if $P(s, y, t, A)=P(s+\theta, y, t+\theta, A)$ for any $0 \leq s<t$, $y \in \mathbb{R}^{m} \times \mathcal{S}$ and $A \in \mathcal{B}\left(\mathbb{R}^{m} \times \mathcal{S}\right)$.

(ii) A family of probability measures $\left\{\mu_{s}, s \geq 0\right\}$ is said to be $\theta$-periodic with respect to the Markov semigroup $\left\{P_{s, t}\right\}$ of $Y(t)$ if

$$
\mu_{s}(A)=\int_{\mathbb{R}^{m} \times \mathcal{S}} P(s, y, s+\theta, A) \mu_{s}(d y), \quad \forall s \geq 0, A \in \mathcal{B}\left(\mathbb{R}^{m} \times \mathcal{S}\right) .
$$

(iii) A stochastic process $\{Y(t), t \geq 0\}$ is said to be a $\theta$-periodic solution of the system given by (2.1) and (2.3) if it is a solution of the system given by (2.1) and (2.3) and is $\theta$-periodic.

Definition 3.2 Let $0 \leq s_{0}<t_{0}<\infty$. A Markovian transition semigroup $\left\{P_{s, t}\right\}$ is said to be regular at $\left(s_{0}, t_{0}\right)$ if all transition probability measures $P\left(s_{0}, y, t_{0}, \cdot\right), y \in \mathbb{R}^{m} \times \mathcal{S}$, are mutually equivalent. $\left\{P_{s, t}\right\}$ is said to be strongly Feller at $\left(s_{0}, t_{0}\right)$ if $P_{s_{0}, t_{0}} f \in C_{b}\left(\mathbb{R}^{m} \times \mathcal{S}\right)$ for any $f \in$ $B_{b}\left(\mathbb{R}^{m} \times \mathcal{S}\right) .\left\{P_{s, t}\right\}$ is said to be irreducible at $\left(s_{0}, t_{0}\right)$ if $P\left(s_{0}, y, t_{0}, A\right)>0$ for any $y \in \mathbb{R}^{m} \times \mathcal{S}$ and any non empty open subset $A$ of $\mathbb{R}^{m} \times \mathcal{S} .\left\{P_{s, t}\right\}$ is said to be regular, strongly Feller, irreducible if it is regular, strongly Feller, irreducible at any $\left(s_{0}, t_{0}\right)$, respectively.

\subsection{Strong Feller and irreducible properties of time-inhomogeneous semigroups}

By Theorem 2.1, under assumptions $\mathbf{A}_{\mathbf{1}}, \mathbf{B}_{\mathbf{1}}$ and $\mathbf{Q}_{\mathbf{1}}$, there exists a unique strong solution $(X(t), \Lambda(t))$ to the hybrid system (2.1) and (2.3). By the interlacing structure, one finds that $(X(t), \Lambda(t))$ is a Markov process on $\mathbb{R}^{m} \times \mathcal{S}$. In this subsection, we will show that the transition semigroup $\left\{P_{s, t}\right\}$ of $(X(t), \Lambda(t))$ is strongly Feller and irreducible.

We make the following assumptions.

A $)$ For each $i \in \mathcal{S}$,

$$
\begin{aligned}
& b(\cdot, 0, i) \in L^{2}\left([0, \theta) ; \mathbb{R}^{m}\right), \sigma(\cdot, 0, i) \in L^{\infty}\left([0, \theta) ; \mathbb{R}^{m}\right), \\
& \int_{\{|u|<1\}}|H(\cdot, 0, i, u)|^{2} \nu(\mathrm{d} u) \in L^{1}\left([0, \theta) ; \mathbb{R}^{m}\right) .
\end{aligned}
$$


For each $n \in \mathbb{N}$, there exists $L_{n} \in L^{\infty}\left([0, \theta) ; \mathbb{R}_{+}\right)$such that for any $t \in[0, \theta), i \in \mathcal{S}$ and $x, y \in \mathbb{R}^{m}$ with $|x| \vee|y| \leq n$,

$$
\begin{aligned}
& |b(t, x, i)-b(t, y, i)|^{2} \leq L_{n}(t)|x-y|^{2}, \quad|\sigma(t, x, i)-\sigma(t, y, i)|^{2} \leq L_{n}(t)|x-y|^{2}, \\
& \int_{\{|u|<1\}}|H(t, x, i, u)-H(t, y, i, u)|^{2} \nu(\mathrm{d} u) \leq L_{n}(t)|x-y|^{2} .
\end{aligned}
$$

A $)$ For each $i \in \mathcal{S}, t \in[0, \theta)$ and $x \in \mathbb{R}^{m}, Q(t, x, i):=\sigma(t, x, i) \sigma^{T}(t, x, i)$ is invertible and

$$
\sup _{|x| \leq n, t \in[0, \theta)}\left|Q^{-1}(t, x, i)\right|<\infty, \quad \forall n \in \mathbb{N}, i \in \mathcal{S} .
$$

$\left.\mathbf{B}_{\mathbf{2}}\right)$ There exists $V_{2} \in C^{1,2}\left(\mathbb{R}_{+} \times \mathbb{R}^{m} ; \mathbb{R}_{+}\right)$such that

$$
\lim _{|x| \rightarrow \infty}\left[\inf _{t \in[0, \infty)} V_{2}(t, x)\right]=\infty
$$

and

$$
\sup _{x \in \mathbb{R}^{m}, t \in[0, \infty)} \mathcal{L}_{i} V_{2}(t, x)<\infty, \quad \forall i \in \mathcal{S}
$$

Obviously, $\mathbf{A}_{\mathbf{2}}$ implies $\mathbf{A}_{\mathbf{1}}$ and $\mathbf{B}_{\mathbf{2}}$ implies $\mathbf{B}_{\mathbf{1}}$.

Theorem 3.3 Suppose that assumptions $\mathbf{A}_{\mathbf{2}}, \mathbf{A}_{\mathbf{3}}, \mathbf{B}_{\mathbf{2}}$ and $\mathbf{Q}_{\mathbf{1}}$ hold. Then the transition semigroup $\left\{P_{s, t}\right\}$ of $(X(t), \Lambda(t))$ is strongly Feller.

Proof. Denote the transition probability function of $(X(t), \Lambda(t))$ by $\{P(s,(x, i), t, B \times\{j\}): 0 \leq$ $\left.s<t,(x, i) \in \mathbb{R}^{m} \times \mathcal{S}, B \in \mathcal{B}\left(\mathbb{R}^{m}\right), j \in \mathcal{S}\right\}$. For $(x, i) \in \mathbb{R}^{m} \times \mathbb{S}$, let $X^{(i)}(t)$ be defined by (2.6). We kill the process $X^{(i)}(t)$ with rate $q_{i}(\cdot)$ and obtain a subprocess $\tilde{X}^{(i)}(t)$ with generator $\mathcal{L}+q_{i i}$. Then,

$$
\mathbb{E}\left[f\left(\widetilde{X}^{(i)}(t)\right)\right]=\mathbb{E}\left[f\left(X^{(i)}(t)\right) \exp \left\{\int_{0}^{t} q_{i i}\left(X^{(i)}(u)\right) d u\right\}\right], \quad f \in \mathcal{B}_{b}\left(\mathbb{R}^{m}\right) .
$$

Let $\widetilde{P}^{(i)}(s, x, \cdot)$ be the transition probability function of $\widetilde{X}^{(i)}(t)$. Then, for $0 \leq s<t, B \in$ $\mathcal{B}\left(\mathbb{R}^{m}\right)$ and $j \in \mathcal{S}$, we have

$$
\begin{aligned}
P(s,(x, i), t, B \times\{j\})= & \delta_{i j} \widetilde{P}^{(i)}(s, x, t, B) \\
& +\int_{s}^{t} \int_{\mathbb{R}^{m}} P\left(t_{1},\left(x_{1}, j_{1}\right), t, B \times\{j\}\right) \sum_{j_{1} \in \mathcal{S} \backslash\{i\}} q_{i j_{1}}\left(x_{1}\right) \widetilde{P}^{(i)}\left(s, x, t_{1}, d x_{1}\right) d t_{1} .
\end{aligned}
$$

Repeating this procedure, we get

$$
P(s,(x, i), t, B \times\{j\})=\delta_{i j} \widetilde{P}^{(i)}(s, x, t, B)+\sum_{k=1}^{n} \Psi_{k}+U_{n},
$$


where

$$
\begin{aligned}
& \Psi_{k}=\int_{s<t_{1}<\cdots<t_{k}<t} \cdots \int_{j_{0}=i, j_{1} \in \mathcal{S} \backslash\left\{j_{0}\right\}, j_{2} \in \mathcal{S} \backslash\left\{j_{1}\right\}, \ldots, j_{k} \in \mathcal{S} \backslash\left\{j_{k-1}\right\}, j_{k}=j} \int_{\mathbb{R}^{m}} \cdots \int_{\mathbb{R}^{m}} \widetilde{P}^{(i)}\left(s, x, t_{1}, d x_{1}\right) \\
& \times q_{i j_{1}}\left(x_{1}\right) \widetilde{P}^{\left(j_{1}\right)}\left(t_{1}, x_{1}, t_{2}, d x_{2}\right) \cdots q_{j_{k-1} j_{k}}\left(x_{k}\right) \widetilde{P}^{\left(j_{k}\right)}\left(t_{k}, x_{k}, t, B\right) d t_{1} d t_{2} \cdots d t_{k},
\end{aligned}
$$

and

$$
\begin{aligned}
& U_{n}= \int_{\substack{s<t_{1}<\cdots<t_{n+1}<t \\
\times j_{j}=i, j_{1} \in \mathcal{S} \backslash\left\{j_{0}\right\}, j_{2} \in \mathcal{S} \backslash\left\{j_{1}\right\}, \ldots, j_{n+1} \in \mathcal{S} \backslash\left\{j_{n}\right\}}} \int_{\mathbb{R}^{m}} \cdots \int_{\mathbb{R}^{m}} \widetilde{P}^{(i)}\left(s, x, t_{1}, d x_{1}\right) \\
& \times q_{i j_{1}}\left(x_{1}\right) \widetilde{P}^{\left(j_{1}\right)}\left(t_{1}, x_{1}, t_{2}, d x_{2}\right) \cdots q_{j_{n} j_{n+1}}\left(x_{n+1}\right) P\left(t_{n+1},\left(x_{n+1}, j_{n+1}\right), t, B \times\{j\}\right) d t_{1} d t_{2} \cdots d t_{n+1} .
\end{aligned}
$$

By the assumption $\mathbf{Q}_{\mathbf{1}}$, we find that $U_{n}$ does not exceed $\frac{[(t-s) L]^{n+1}}{(n+1) !}$. Hence

$$
P(s,(x, i), t, B \times\{j\})=\delta_{i j} \widetilde{P}^{(i)}(s, x, t, B)+\sum_{n=1}^{\infty} \Psi_{n} .
$$

By [4, Theorem 3.8] and assumptions $\mathbf{A}_{\mathbf{2}}, \mathbf{A}_{\mathbf{3}}, \mathbf{B}_{\mathbf{2}}$, we know that the transition semigroup of $X^{(i)}(t)$ is strongly Feller. Following the argument of [15, Lemma 4.5], we can show that the transition semigroup of $\widetilde{X}^{(i)}(t)$ is also strongly Feller. Then, $\widetilde{P}^{(i)}(s, x, t, B)$ and $\Psi_{n}, n \in \mathbb{N}$, are all continuous with respect to $x$. Note that $\mathcal{S}$ is equipped with a discrete metric. Then, the left-hand side of (3.3) is lower semi-continuous with respect to $(x, i)$. Therefore, the transition semigroup $\left\{P_{s, t}\right\}$ of $(X(t), \Lambda(t))$ is strongly Feller by [9, Proposition 6.1.1].

Now we consider the irreducibility of the transition semigroup $\left\{P_{s, t}\right\}$ of $(X(t), \Lambda(t))$. Denote by $B_{b, l o c}\left(\mathbb{R}_{+}\right)$and $B_{b, l o c}\left([0, \infty) \times \mathbb{R}^{m} ; \mathbb{R}^{m}\right)$ the sets of all locally bounded Borel measurable functions on $\mathbb{R}_{+}$and maps from $[0, \infty) \times \mathbb{R}^{m}$ to $\mathbb{R}^{m}$, respectively. Let $f$ be a function on $[0, \infty) \times \mathbb{R}^{m}$. For $\rho>0$, we define

$$
f^{\bullet \rho}(t, x)=f(t, \rho x), \quad t \geq 0, x \in \mathbb{R}^{m} .
$$

We make the following assumptions.

$\left.\mathbf{B}_{3}\right)$ There exists $V_{3} \in C^{1,2}\left([0, \infty) \times \mathbb{R}^{m} ; \mathbb{R}_{+}\right)$satisfying the following conditions:

$$
\lim _{|x| \rightarrow \infty}\left[\inf _{t \in[0, \infty)} V_{3}(t, x)\right]=\infty
$$

(ii) For any $\rho \geq 1$, there exist $q_{\rho} \in B_{b, l o c}\left(\mathbb{R}_{+}\right)$and $W_{\rho}(t, x) \in B_{b, l o c}\left([0, \infty) \times \mathbb{R}^{m} ; \mathbb{R}^{m}\right)$ satisfying for each $n \in \mathbb{N}$ there exists $R_{n} \in L_{\text {loc }}^{1}\left([0, \infty) ; \mathbb{R}_{+}\right)$such that for any $t \in[0, \infty)$ and $x, y \in \mathbb{R}^{m}$ with $|x| \vee|y| \leq n$,

$$
\left|W_{\rho}(t, x)-W_{\rho}(t, y)\right|^{2} \leq R_{n}(t)|x-y|^{2},
$$

and for $t \geq 0, x \in \mathbb{R}^{m}$ and $i \in \mathcal{S}$,

$$
\mathcal{L}_{i} V_{3}^{\bullet \rho}(t, x) \leq q_{\rho}(t)
$$




$$
V_{3}^{\bullet \rho}(t, x) \leq\left\langle W_{\rho}, \nabla_{x} V_{3}^{\bullet \rho}(t, x)\right\rangle
$$

$\left.\mathbf{Q}_{\mathbf{2}}\right)$ For any distinct $i, j \in \mathcal{S}$, there exist $j_{1}, \ldots, j_{r} \in \mathcal{S}$ with $j_{i} \neq j_{i+1}, j_{1}=i$ and $j_{r}=j$ such that the set $\left\{x \in \mathbb{R}^{m}: q_{j_{i} j_{i+1}}(x)>0\right\}$ has positive Lebesgue measure for $i=1, \ldots, r$.

Theorem 3.4 Suppose that assumptions $\mathbf{A}_{\mathbf{1}}, \mathbf{A}_{\mathbf{3}}, \mathbf{B}_{\mathbf{1}}, \mathbf{B}_{\mathbf{3}}, \mathbf{Q}_{\mathbf{1}}$ and $\mathbf{Q}_{\mathbf{2}}$ hold. Then, the transition semigroup $\left\{P_{s, t}\right\}$ of $(X(t), \Lambda(t))$ is irreducible.

Proof. Denote the transition probability function of $(X(t), \Lambda(t))$ by $\{P(s,(x, i), t, B \times\{j\}): 0 \leq$ $\left.s<t,(x, i) \in \mathbb{R}^{m} \times \mathcal{S}, B \in \mathcal{B}\left(\mathbb{R}^{m}\right), j \in \mathcal{S}\right\}$. As shown in the proof Theorem 3.3, the assumption $\mathrm{Q}_{1}$ implies that (3.3) holds. By [4, Theorem 3.3.9], we know that for any $i \in \mathcal{S}$, the transition semigroup of $X^{(i)}(t)$ is irreducible under assumptions $\mathbf{A}_{\mathbf{1}}, \mathbf{A}_{\mathbf{3}}, \mathbf{B}_{\mathbf{1}}$ and $\mathbf{B}_{\mathbf{3}}$. Further, we find that the subprocess $\widetilde{X}^{(i)}(t)$ is irreducible by the assumption $\mathbf{Q}_{\mathbf{1}}$. This together with the assumption $\mathrm{Q}_{2}$ implies that the right-hand side of (3.3) is positive whenever $B$ is a non empty open set of $\mathbb{R}^{m}$. Then, $P(s,(x, i), t, B \times\{j\})>0$. Since $B$ is arbitrary, the transition semigroup $\left\{P_{s, t}\right\}$ of $(X(t), \Lambda(t))$ is irreducible.

\subsection{Existence and uniqueness of periodic solutions}

To prove the existence and uniqueness of periodic solutions to the hybrid system (2.1) and (2.3), we make the following assumptions.

$\left.\mathrm{Q}_{3}\right)$

$$
\sum_{j=1}^{\infty} j \sup _{i \in \mathcal{S} \backslash\{j\}, x \in \mathbb{R}^{m}} q_{i j}(x)<\infty .
$$

$\left.\mathbf{B}_{4}\right)$ There exists $V \in C^{1,2}\left([0, \infty) \times \mathbb{R}^{m} ; \mathbb{R}_{+}\right)$satisfying the following conditions:

$$
\lim _{|x| \rightarrow \infty}\left[\inf _{t \in[0, \infty)} V(t, x)\right]=\infty
$$

and

$$
\lim _{n \rightarrow \infty} \sup _{|x|>n, i \in \mathcal{S}, t \in[0, \infty)} \mathcal{L}_{i} V(t, x)=-\infty
$$

(ii) For any $\rho \geq 1$, there exist $q_{\rho} \in B_{b, l o c}\left(\mathbb{R}_{+}\right)$and $W_{\rho}(t, x) \in B_{b, l o c}\left([0, \infty) \times \mathbb{R}^{m} ; \mathbb{R}^{m}\right)$ satisfying for each $n \in \mathbb{N}$ there exists $R_{n} \in L_{l o c}^{1}\left([0, \infty) ; \mathbb{R}_{+}\right)$such that for any $t \in[0, \infty)$ and $x, y \in \mathbb{R}^{m}$ with $|x| \vee|y| \leq n$

$$
\left|W_{\rho}(t, x)-W_{\rho}(t, y)\right|^{2} \leq R_{n}(t)|x-y|^{2}
$$

and

$$
\begin{gathered}
\sup _{x \in \mathbb{R}^{m}, i \in \mathcal{S}, t \in[0, \infty)} \mathcal{L}_{i} V^{\bullet \rho}(t, x)<\infty, \\
V^{\bullet \rho}(t, x) \leq\left\langle W_{\rho}, \nabla_{x} V^{\bullet \rho}(t, x)\right\rangle, \quad \forall t \geq 0, x \in \mathbb{R}^{m} .
\end{gathered}
$$

Obviously, $\mathbf{Q}_{\mathbf{3}}$ implies $\mathbf{Q}_{\mathbf{1}}$ and $\mathbf{B}_{\mathbf{4}}$ implies $\mathbf{B}_{\mathbf{1}}-\mathbf{B}_{\mathbf{3}}$. 
Theorem 3.5 Suppose that assumptions $\mathbf{A}_{\mathbf{2}}, \mathbf{A}_{\mathbf{3}}, \mathbf{B}_{\mathbf{4}}, \mathbf{Q}_{\mathbf{2}}$ and $\mathbf{Q}_{\mathbf{3}}$ hold. Then,

(i) The hybrid system given by (2.1) and (2.3) has a unique $\theta$-periodic solution $(X(t), \Lambda(t))$.

(ii) The Markovian transition semigroup $\left\{P_{s, t}\right\}$ of $(X(t), \Lambda(t))$ is strongly Feller and irreducible.

(iii) Let $\mu_{s}(A)=\mathbb{P}((X(t), \Lambda(t)) \in A)$ for $A \in \mathcal{B}\left(\mathbb{R}^{m} \times \mathcal{S}\right)$ and $s \geq 0$. Then, for any $s \geq 0$ and $\varphi \in L^{2}\left(\mathbb{R}^{m} \times \mathcal{S} ; \mu_{s}\right)$, we have

$$
\lim _{n \rightarrow \infty} \frac{1}{n} \sum_{i=1}^{n} P_{s, s+i \theta} \varphi=\int_{\mathbb{R}^{m} \times \mathcal{S}} \varphi d \mu_{s} \text { in } L^{2}\left(\mathbb{R}^{m} \times \mathcal{S} ; \mu_{s}\right) .
$$

Proof. Let $(X(t), \Lambda(t))$ be the unique strong solution to the hybrid system given by (2.1) and (2.3) with initial value $(x, i) \in \mathbb{R}^{m} \times \mathcal{S}$. Define

$$
\widetilde{V}(t, x, i)=V(t, x)+i, \quad t \geq 0, x \in \mathbb{R}^{m}, i \in \mathcal{S},
$$

where $V$ is given in the assumption $\mathbf{B}_{\mathbf{4}}$. Note that

$$
\mathcal{A} \widetilde{V}(t, x, i)=\mathcal{L}_{i} V(t, x)+\sum_{j \in \mathcal{S} \backslash\{i\}} q_{i j}(x)(j-i) .
$$

Then, we obtain by assumptions $\mathbf{B}_{\mathbf{4}}$ and $\mathbf{Q}_{\mathbf{3}}$ that

$$
\begin{aligned}
& \lim _{|x|+i \rightarrow \infty}\left[\inf _{t \in[0, \infty)} \tilde{V}(t, x, i)\right]=\infty, \\
& \lim _{n \rightarrow \infty} \sup _{|x|+i>n, t \in[0, \infty)} \mathcal{A} \widetilde{V}(t, x, i)=-\infty
\end{aligned}
$$

and

$$
\sup _{x \in \mathbb{R}^{m}, i \in \mathcal{S}, t \in[0, \infty)} \mathcal{A} \widetilde{V}(t, x, i)<\infty .
$$

For $n \in \mathbb{N}$, we define the stopping time $T_{n}$ by

$$
T_{n}=\inf \{t \in[0, \infty):|X(t)| \vee \Lambda(t) \geq n\} .
$$

For $t \geq 0$, by Itô's formula, we get

$$
\mathbb{E}\left[\widetilde{V}\left(t \wedge T_{n}, X\left(t \wedge T_{n}\right), \Lambda\left(t \wedge T_{n}\right)\right)\right]=\mathbb{E}[\widetilde{V}(0, X(0), \Lambda(0))]+\mathbb{E}\left[\int_{0}^{t \wedge T_{n}} \mathcal{A} \widetilde{V}(u, X(u), \Lambda(u)) \mathrm{d} u\right]
$$

Define

$$
A_{n}:=-\sup _{|y|+k>n, t \in[0, \infty)} \mathcal{A} \tilde{V}(t, y, k) .
$$

By (3.5), we get

$$
\lim _{n \rightarrow \infty} A_{n}=+\infty
$$


We have

$$
\mathcal{A} \widetilde{V}(u, X(u), \Lambda(u)) \leq-1_{\{|X(u)|+k \geq n\}} A_{n}+\sup _{|y|+k<n, u \in[0, \infty)} \mathcal{A} \widetilde{V}(u, y, k) .
$$

Then, there exist positive constants $c_{1}$ and $c_{2}$ such that for large $n$,

$$
\mathbb{E}\left[\int_{0}^{t \wedge T_{n}} 1_{\{|X(u)|+k \geq n\}} \mathrm{d} u\right] \leq \frac{c_{1} t+c_{2}}{A_{n}} .
$$

Denote $B_{n}=\left\{(y, k) \in \mathbb{R}^{m} \times \mathcal{S}:|y|+k<n\right\}$ and $B_{n}^{c}=\left\{(y, k) \in \mathbb{R}^{m} \times \mathcal{S}:|y|+k \geq n\right\}$. Letting $n \rightarrow \infty$ in (3.9), we obtain by (3.8) that

$$
\lim _{n \rightarrow \infty} \varlimsup_{T \rightarrow \infty} \frac{1}{T} \int_{0}^{T} P\left(0,(x, i), u, B_{n}^{c}\right) \mathrm{d} u=0 .
$$

By (3.6), there exists $\lambda>0$ such that

$$
\mathcal{A} \widetilde{V}(t, x, i) \leq \lambda, \quad \forall t \geq 0,(x, i) \in \mathbb{R}^{m} \times \mathcal{S} .
$$

By (3.7) and (3.11), we get

$$
\mathbb{E}[\widetilde{V}(t, X(t), \Lambda(t))] \leq \lambda t+\widetilde{V}(0, x, i)
$$

Together with Chebyshev's inequality, this implies that

$$
\mathbb{P}\left(0,(x, i), t, B_{n}^{c}\right) \leq \frac{\lambda t+\widetilde{V}(0, x, i)}{\inf _{|y|+k>n, t \in[0, \infty)} \widetilde{V}(t, y, k)} .
$$

By (3.4) and (3.12), we find that there exists a sequence of positive integers $\gamma_{n} \uparrow \infty$ such that

$$
\lim _{n \rightarrow \infty}\left\{\sup _{(x, i) \in B_{\gamma_{n}}, t \in(0, \theta)} \mathbb{P}\left(0,(x, i), t, B_{n}^{c}\right)\right\}=0 .
$$

Similar to [4, Lemma 3.10], we can show that the transition semigroup $\left\{P_{s, t}\right\}$ of the Markov process $(X(t), \Lambda(t))$ is $\theta$-periodic. Combining this with (3.10), (3.13) and [6. Theorem 3.2 and Remark 3.1], we conclude that the hybrid system given by (2.1) and (2.3) has a $\theta$-periodic solution. Here we would like to call the reader's attention to a missing condition in [6, Theorem 3.2], which was pointed out by $\mathrm{Hu}$ and $\mathrm{Xu}$ recently. According to $\mathrm{Hu}$ and $\mathrm{Xu}$ [5, Theorem 2.1 and Remark A.1], [6, Theorem 3.2 and Remark 3.1] holds under the additional assumption that $\left\{P_{s, t}\right\}$ is a Feller semigroup. By Theorem [3.3, $\left\{P_{s, t}\right\}$ is a strong Feller semigroup and hence we can apply [6. Theorem 3.2 and Remark 3.1] to show that the hybrid system given by (2.1) and (2.3) has a $\theta$-periodic solution.

By using the same argument, we can show that [4, Lemmas 3.12 and 3.13] hold with the state space $\mathbb{R}^{m}$ replaced by $\mathbb{R}^{m} \times \mathcal{S}$. Hence the uniqueness of the $\theta$-periodic solution is a direct consequence of Theorem 3.3 and Theorem 3.4. Finally, the last assertion of the theorem can be proved by following the same argument of the proof of [4, Lemma 3.13]. 


\section{$4 \quad$ Examples}

In this section, we use two examples to illustrate our main results.

Example 4.1 (Stochastic Lorenz equation with regime switching)

We consider the following stochastic Lorenz equation ([7]) with regime switching:

$$
\begin{aligned}
d X_{1}(t)= & {\left[-\alpha(t, \Lambda(t)) X_{1}(t)+\alpha(t, \Lambda(t)) X_{2}(t)\right] d t+\sum_{j=1}^{3} \sigma_{1 j}(t, X(t), \Lambda(t)) d B_{j}(t) } \\
& +\int_{\{|u|<1\}} H_{1}(t, X(t-), \Lambda(t-), u) \tilde{N}(d t, d u)+\int_{\{|u| \geq 1\}} G_{1}(t, X(t-), \Lambda(t-), u) N(d t, d u), \\
d X_{2}(t)= & {\left[\mu(t, \Lambda(t)) X_{1}(t)-X_{2}(t)-X_{1}(t) X_{3}(t)\right] d t+\sum_{j=1}^{3} \sigma_{2 j}(t, X(t), \Lambda(t)) d B_{j}(t) } \\
& +\int_{\{|u|<1\}} H_{2}(t, X(t-), \Lambda(t-), u) \tilde{N}(d t, d u)+\int_{\{|u| \geq 1\}} G_{2}(t, X(t-), \Lambda(t-), u) N(d t, d u), \\
d X_{3}(t)= & {\left[-\beta(t, \Lambda(t)) X_{3}(t)+X_{1}(t) X_{2}(t)\right] d t+\sum_{j=1}^{3} \sigma_{3 j}(t, X(t), \Lambda(t)) d B_{j}(t) } \\
& +\int_{\{|u|<1\}} H_{3}(t, X(t-), \Lambda(t-), u) \tilde{N}(d t, d u)+\int_{\{|u| \geq 1\}} G_{3}(t, X(t-), \Lambda(t-), u) N(d t, d u),
\end{aligned}
$$

where $\Lambda(t)$ takes values in $\mathcal{S}=\{1,2, \ldots\}$ and is generated by $Q=\left(q_{i j}(x)\right)$ that satisfies assumptions $\mathbf{Q}_{\mathbf{2}}$ and $\mathbf{Q}_{\mathbf{3}}, \alpha(t, i), \beta(t, i), \mu(t, i):[0, \infty) \times \mathcal{S} \rightarrow \mathbb{R}_{+}, \sigma(t, x, i):[0, \infty) \times \mathbb{R}^{3} \times \mathcal{S} \rightarrow \mathbb{R}^{3 \times 3}$, $H(t, x, i, u):[0, \infty) \times \mathbb{R}^{3} \times \mathcal{S} \times \mathbb{R}^{l} \rightarrow \mathbb{R}^{3}$ and $G(t, x, i, u):[0, \infty) \times \mathbb{R}^{3} \times \mathcal{S} \times \mathbb{R}^{l} \rightarrow \mathbb{R}^{3}$ are all Borel measurable and periodic w.r.t. $t$ with period $\theta$.

We assume that $\sigma(t, x, i)$ and $H(t, x, i, u)$ satisfy (3.1) and (3.2), and there exist $\gamma>0$ and a continuously differentiable periodic function $a(t)$ with period $\theta$ such that for any $t \in[0, \theta), i \in \mathcal{S}$,

$$
\gamma<\alpha(t, i), \beta(t, i), \mu(t, i) \leq a(t) .
$$

Moreover, for any $\varepsilon>0$ there exists $c_{\varepsilon}>0$ such that for any $i \in \mathcal{S}$,

$$
|\sigma(t, x, i)|^{2}+\int_{\{|u|<1\}}|H(t, x, i, u)|^{2} \nu(d u)+\int_{\{|u| \geq 1\}}|G(t, x, i, u)|^{2} \nu(d u) \leq \varepsilon|x|^{2}+c_{\varepsilon} .
$$

Define

$$
V(t, x)=x_{1}^{2}+x_{2}^{2}+\left(x_{3}-2 a(t)\right)^{2} .
$$

We will show that $V$ satisfies the assumption $\mathbf{B}_{\mathbf{4}}$. Without loss of generality, we assume that $\rho=1$. The verification for the case that $\rho>1$ is completely similar. Let

$$
W(t, x)=\frac{1}{2}\left(x_{1}, x_{2}, x_{3}-2 a(t)\right)
$$


Then,

$$
V(t, x)=\left\langle W, V_{x}(t, x)\right\rangle
$$

For $\varepsilon>0$ and $i \in \mathcal{S}$, we obtain by (4.2) that

$$
\begin{aligned}
\mathcal{L}_{i} V(t, x) \leq & 4\left(2 a(t)-x_{3}\right) a^{\prime}(t)-2 \alpha(t, i) x_{1}^{2}-2 x_{2}^{2}-2 \beta(t, i)\left[x_{3}^{2}-2 a(t) x_{3}\right]+|\sigma(t, x, i)|^{2} \\
& +\sum_{j=1}^{3} \int_{\{|u|<1\}}\left|H_{j}(t, x, i, u)\right|^{2} \nu(d u)+\sum_{j=1}^{2} \int_{\{|u| \geq 1\}}\left[\left(x_{j}+G_{j}(t, x, i, u)\right)^{2}-x_{j}^{2}\right] \nu(d u) \\
& +\int_{\{|u| \geq 1\}}\left[\left(x_{3}+G_{3}(t, x, u)-2 a(t)\right)^{2}-\left(x_{3}-2 a(t)\right)^{2}\right] \nu(d u) \\
\leq & 4\left(2 a(t)+\left|x_{3}\right|\right)\left|a^{\prime}(t)\right|-2\left[\gamma x_{1}^{2}+x_{2}^{2}+\gamma x_{3}^{2}-2(a(t))^{2}\left|x_{3}\right|\right]+\varepsilon|x|^{2}+c_{\varepsilon} \\
& +2\left[\left|x_{1}\right|+\left|x_{2}\right|+\left|x_{3}\right|+2 a(t)\right][\nu(\{|u| \geq 1\})]^{1 / 2}\left(\varepsilon|x|^{2}+c_{\varepsilon}\right)^{1 / 2} .
\end{aligned}
$$

Then,

$$
\lim _{n \rightarrow \infty} \sup _{|x|>n, i \in \mathcal{S}, t \in[0, \infty)} \mathcal{L}_{i} V(t, x)=-\infty, \quad \sup _{x \in \mathbb{R}^{m}, i \in \mathcal{S}, t \in[0, \infty)} \mathcal{L}_{i} V(t, x)<\infty .
$$

Thus, the assumption $\mathbf{B}_{\mathbf{4}}$ is satisfied. Therefore, the SDE (4.1) has a unique $\theta$-periodic solution $(X(t), \Lambda(t))$ and assertions (ii) and (iii) of Theorem 3.5 hold.

Example 4.2 (Stochastic equation of the lemniscate of Bernoulli with regime switching)

In this example, we consider the stochastic equation of the lemniscate of Bernoulli, which generalizes [1, Example 3.20] to the non-autonomous case with Lévy noise and regime-switching.

For $x=\left(x_{1}, x_{2}\right) \in \mathbb{R}^{2}$, define

$$
I(x)=\left(x_{1}^{2}+x_{2}^{2}\right)^{2}-4\left(x_{1}^{2}-x_{2}^{2}\right) .
$$

Let

$$
\mathcal{V}(I)=\frac{I^{2}}{2\left(1+I^{2}\right)^{3 / 4}}, \quad \mathcal{H}(I)=\frac{I}{\left(1+I^{2}\right)^{3 / 8}} .
$$

Consider the vector field

$$
b(x)=-\left[\mathcal{V}_{x}(I)+\left(\frac{\partial \mathcal{H}(I)}{\partial x_{2}},-\frac{\partial \mathcal{H}(I)}{\partial x_{1}}\right)^{T}\right]
$$

We have

$$
\begin{aligned}
& \frac{d \mathcal{V}(I)}{d I}=\frac{I\left(I^{2}+4\right)}{4\left(1+I^{2}\right)^{7 / 4}}, \quad \frac{d \mathcal{H}(I)}{d I}=\frac{I^{2}+4}{4\left(1+I^{2}\right)^{11 / 4}} \\
& \frac{\partial I}{\partial x_{1}}=4 x_{1}\left(x_{1}^{2}+x_{2}^{2}\right)-8 x_{1}, \quad \frac{\partial I}{\partial x_{2}}=4 x_{2}\left(x_{1}^{2}+x_{2}^{2}\right)+8 x_{2} .
\end{aligned}
$$

Define

$$
f(I)=\frac{d \mathcal{V}(I)}{d I}, \quad g(I)=\frac{d \mathcal{H}(I)}{d I}
$$


Then,

$$
\mathcal{V}_{x}(I)=\frac{d \mathcal{V}(I)}{d I}\left(\frac{\partial I}{\partial x_{1}}, \frac{\partial I}{\partial x_{2}}\right)^{T}
$$

and

$$
\begin{aligned}
& b_{1}(x)=-f(I)\left(4 x_{1}\left(x_{1}^{2}+x_{2}^{2}\right)-8 x_{1}\right)-g(I)\left(4 x_{2}\left(x_{1}^{2}+x_{2}^{2}\right)+8 x_{2}\right), \\
& b_{2}(x)=-f(I)\left(4 x_{2}\left(x_{1}^{2}+x_{2}^{2}\right)+8 x_{2}\right)-g(I)\left(-4 x_{1}\left(x_{1}^{2}+x_{2}^{2}\right)+8 x_{1}\right) .
\end{aligned}
$$

We consider the following SDEs:

$$
\begin{aligned}
d X_{1}(t)= & b_{1}(X(t)) d t+\sigma_{11}(t, X(t), \Lambda(t)) d B_{1}(t)+\sigma_{12}(t, X(t), \Lambda(t)) d B_{2}(t) \\
& +\int_{\{|u|<1\}} H_{1}(t, X(t-), \Lambda(t-), u) \tilde{N}(d t, d u)+\int_{\{|u| \geq 1\}} G_{1}(t, X(t-), \Lambda(t-), u) N(d t, d u), \\
d X_{2}(t)= & b_{2}(X(t)) d t+\sigma_{21}(t, X(t), \Lambda(t)) d B_{1}(t)+\sigma_{22}(t, X(t), \Lambda(t)) d B_{2}(t) \\
& +\int_{\{|u|<1\}} H_{2}(t, X(t-), \Lambda(t-), u) \tilde{N}(d t, d u)+\int_{\{|u| \geq 1\}} G_{2}(t, X(t-), \Lambda(t-), u) N(d t, d u),
\end{aligned}
$$

where $\Lambda(t)$ takes values in $\mathcal{S}=\{1,2 \ldots\}$ and is generated by $Q(x)=\left(q_{i j}(x)\right)$ with

$$
q_{i j}(x)=\frac{1 \wedge|x|}{j^{2+\delta}} \text { for some } \delta>0
$$

$\sigma(t, x, i):[0, \infty) \times \mathbb{R}^{2} \times \mathcal{S} \rightarrow \mathbb{R}^{2 \times 2}, H(t, x, i, u):[0, \infty) \times \mathbb{R}^{2} \times \mathcal{S} \times \mathbb{R}^{l} \rightarrow \mathbb{R}^{2}$ and $G(t, x, i, u):$ $[0, \infty) \times \mathbb{R}^{2} \times \mathcal{S} \times \mathbb{R}^{l} \rightarrow \mathbb{R}^{2}$ are all Borel measurable and periodic w.r.t. $t$ with period $\theta$. We assume that $\sigma(t, x, i)$ and $H(t, x, i, u)$ satisfy (3.1) and (3.2). Moreover, for any $\varepsilon>0$, there exists $c_{\varepsilon}>0$ such that for any $i \in \mathcal{S}$,

$$
\begin{gathered}
|\sigma(t, x, i)|^{2}+\int_{\{|u|<1\}}|H(t, x, i, u)|^{2} \nu(d u)+|x| \int_{\{|u| \geq 1\}}|G(t, x, i, u)| \nu(d u) \\
+\int_{\{|u| \geq 1\}}|G(t, x, i, u)|^{2} \nu(d u) \leq \varepsilon|x|^{2}+c_{\varepsilon} .
\end{gathered}
$$

Define

$$
V(t, x)=\mathcal{V}(I(x)) .
$$

Following the argument of [4, Example 4.3], we can check that the assumption $\mathbf{B}_{\mathbf{4}}$ is satisfied. Therefore, the SDE (4.3) has a unique $\theta$-periodic solution $(X(t), \Lambda(t))$ and assertions (ii) and (iii) of Theorem 3.5 hold. 


\section{References}

[1] Chen L., Dong Z., Jiang J. and Zhai J., On limiting behavior of stationary measures for stochastic evolution systems with small noise intensity. Sci. China Math. 63 (2020) 1463-1504.

[2] Chen X., Chen Z., Tran K. and Yin G., Properties of switching jump diffusions: maximum principles and Harnack inequalities. Bernoulli 25 (2019) 1045-1075.

[3] Chen X., Chen Z., Tran K. and Yin G., Recurrence and ergodicity for a class of regimeswitching jump diffusions. Appl. Math. Optim. 80 (2019) 415-445.

[4] Guo X. and Sun W., Periodic solutions of stochastic differential equations driven by Lévy noises. https://arxiv.org/pdf/1905.04843v2.pdf.

[5] $\mathrm{Hu} \mathrm{H}$. and Xu L., Existence and uniqueness theorems for periodic Markov process and applications to stochastic functional differential equations. J. Math. Anal. Appl. 466 (2018) 896-926.

[6] Khasminskii R.Z., Stochastic Stability of Differential Equations. Springer-Verlag, Second Edition, 2012.

[7] Lorenz E.N., Deterministic nonperiodic flow. J. Atmos. Sci. 20 (1963) 130-141.

[8] Mao X. and Yuan C., Stochastic Differential Equations with Markovian Switching. Imperial College Press, London, 2006.

[9] Meyn S.P. and Tweedie R.L., Markov Chains and Stochastic Stability. Communications and Control Engineering Series. Springer-Verlag London, 1993.

[10] Nguyen D.H. and Yin G., Modeling and analysis of switching diffusion systems: pastdependent switching with a countable state space. SIAM J. Control Optim. 54 (2016) $2450-2477$.

[11] Nguyen D.H. and Yin G., Recurrence and ergodicity of switching diffusions with pastdependent switching having a countable state space. Potent. Anal. 48 (2018) 405-435.

[12] Shao J., Strong solutions and strong Feller properties for regime-switching diffusion processes in an infinite state space. SIAM J. Control Optim. 4 (2015) 2462-2479.

[13] Xi F., Asymptotic properties of jump-diffusion processes with state-dependent switching. Stoch. Proc. Appl. 119 (2009) 2198-2221.

[14] Xi F. and Yin G., Jump-diffusions with state-dependent switching: existence and uniqueness, Feller property, linearization, and uniform ergodicity. Sci. China Math. 12 (2011) 2651-2667.

[15] Xi F., Yin G. and Zhu C., Regime-switching jump diffusions with non-Lipschitz coefficients and countably many switching states: existence and uniqueness, Feller, and strong Feller properties. Modeling, Stochastic Control, Optimization, and Applications. 571-599, 2019. 
[16] Xi F. and Zhu C., On Feller and strong Feller properties and expeniential ergodicity of regime-switching jump diffusion processes with countable regimes. SIAM J. Control. Optim. 55 (2017) 1789-1818.

[17] Yin G. and Zhu C., Hybrid Switching Diffusions: Properties and Applications. Stochastic Medelling and Applied Probability. Vol. 63. Springer, New York, 2010.

[18] Zhang X., Wang K. and Li D., Stochastic periodic solutions of stochastic differential equations driven by Lévy process. J. Math. Anal. Appl. 430 (2015) 231-242. 